\title{
Test and device to evaluate the capillary absorption in soil specimens of adobe and rammed earth
}

\author{
Silvia Augusta Cirvini, J.A. Gómez Voltan \\ INCIHUSA CONICET (Consejo Nacional de Investigaciones Científicas y Técnicas de Argentina). \\ Av. Ruiz Leal s/n Parque General San Martín. Código Postal 5500. Mendoza, Argentina \\ Fax: 0542615244001 Telf: 0542615244324 \\ scirvini@mendoza-conicet.gob.ary gvoltan@mendoza-conicet.gob.ar
}

\begin{abstract}
The moisture degree of capillary rise is an indicator of the state of conservation of earthen construction and their mechanical response to load cases, especially seismic action, since the buildings diminish resistant capacity where they are more demanding by the base shear. This paper shows a test procedure and a specific device to evaluate the rate of rise and variation of moisture content in samples of soil for use in new or existing walls of adobe and tapia. These parameters allow evaluating the suitability of building material, the improvements efficiency to increase its quality (modification of its texture, compactness, and composition), choice and dosage of additives for moisture control. In addition, in case of patrimonial buildings affected by rising damp, allows to design and to prove procedures for structural rehabilitation, and using them in the building after evaluating his efficiency and scope.
\end{abstract}

Keywords: device absorption test, water absorption test, adobe, rammed earth, soil, capillary rise.

\section{Ensayo y dispositivo para evaluar la absorción capilar en probetas de adobe y tapia}

\section{Resumen}

El grado de humedad derivada del ascenso capilar es un indicador del estado de conservación de las construcciones de tierra y por ende de su respuesta mecánica ante diversas solicitaciones, en particular bajo la acción sísmica, pues las edificaciones disminuyen su capacidad resistente justamente donde se encuentran más exigidas por el corte basal. El resultado de esta etapa del estudio de la humedad capilar, ha sido la concreción de un procedimiento de ensayo y un dispositivo específico para evaluar la velocidad de ascenso y la variación del contenido de humedad en probetas de suelo alteradas destinado a su uso en obra nueva o inalteradas extraídas de muros existentes de adobe y tapia. El conocimiento de estos parámetros permite evaluar la aptitud del material en la edificación, la eficiencia de mejoras introducidas (modificación de su textura, compacidad, composición), y la elección de aditivos y sus dosificaciones para el control de la humedad. Además, en el caso de edificios histórico-patrimoniales afectados por esta patología, nos permite diseñar y probar diferentes procedimientos para rehabilitación estructural, aplicándolos a la construcción una vez conocidos su eficiencia y alcance.

Palabras clave: ensayo, dispositivo, absorción capilar, adobe, tapia, suelo, humedad capilar. 


\section{Introducción}

En el marco de un proyecto "Bienes culturales y Desarrollo local. Bases para un plan de manejo del Patrimonio Cultural Ambiental del Área metropolitana de Mendoza, con vistas al aprovechamiento turístico sustentable y la rehabilitación integral de sus localidades" se relevaron más de 1200 edificios patrimoniales. Se trató de un proyecto trianual de investigación y transferencia desarrollado por nuestro equipo entre 2008 y 2010, con financiamiento de la Agencia Nacional de Promoción Científica y Tecnológica y CONICET de Argentina.

El procesamiento estadístico de los datos obtenidos permite afirmar que el 37\% de los edificios históricos evaluados en la región de Cuyo-Argentina, son parcial o totalmente de adobe, tapia o quincha, de los cuales el $96 \%$ de ellos se encuentran afectados por humedad ascendente desde la subestructura. La altura alcanzada, medida desde el solado, varía entre $20 \mathrm{~cm}$ y $120 \mathrm{~cm}$, además, junto a esta patología se observan los típicos daños que conlleva: revoques sin adherencia al sustrato, desmenuzamientos, eflorescencias, pintura descascarada, etc. [1].

Conocido es que el mecanismo de ascenso capilar se basa en la tensión superficial de los líquidos que le permite ascender en materiales porosos por sobre su nivel freático venciendo la fuerza de gravedad. El agua presenta altos valores de tensión superficial $(\gamma=73 \mathrm{mN} / \mathrm{m})$. Si la subestructura de la edificación no tiene una adecuada resolución de aislación hidráulica, permitirá que el agua capilar suba por dentro del muro hasta que el peso de la columna de agua se equilibre con la fuerza de ascenso generada por la tensión superficial. Luego, en busca de un equilibrio higroscópico, el muro intercambia humedad con el ambiente.

El suelo empleado en construcción presenta en general, una matriz conformada por material grueso o incoherente y arcillas, una interpretación simplista asigna al primero la capacidad portante y a las arcillas un efecto aglutinante y estructurador del material grueso. Las propiedades electroquímicas presente en los minerales de las arcillas dan origen a la cohesión y plasticidad del material. Las tres fases observables en un suelo (sólido, líquido y gaseoso), le otorgan el carácter de "poroso" que facilita el ascenso capilar del agua.
Esta agua intersticial interfiere el equilibrio electrostático de la matriz y además genera un importante trabajo mecánico en el umbral que separa la zona saturada y no-saturada, y entre ésta y la "seca". Estos umbrales (vadose zone) varían su posición en función de la freática y en particular de la humedad relativa del ambiente. La combinación de estos efectos genera reducción de los valores de cohesión, de la capacidad portante del muro y una disgregación progresiva del material que se acentúa en las zonas de interfase. Claro es entonces, que el ascenso capilar en muros es una patología importante, y ciertamente peligrosa en un entorno de alta sismicidad.

Por lo dicho, surge la necesidad de un estudio científico y crítico del mecanismo de ascenso del agua en suelo usado en muros. La mayoría de los trabajos existentes se basan en recetas aplicables al lugar y tipo de suelo para el que fueron propuestos y aún así son de relativa o cuestionable eficacia, pues en general, se desconoce la evolución en el tiempo de dichas intervenciones [2].

Si bien actualmente se ha encarado este estudio a nivel mundial en un entorno científico y de laboratorio, este mecanismo en estructuras de tierra, no ha sido descripto totalmente, y todavía no se han aportado soluciones al campo de la restauración y la consolidación estructural. Entre las instituciones que han abordado científicamente el problema de la humedad capilar tanto en obras patrimoniales como en construcción nueva se encuentran CRATERRE, la Universidad Politécnica de Madrid, The Getty Conservation Institute, el Instituto Torroja y la Universidad de Kassel.

El Laboratorio del INCIHUSA Conicet, CCT Mendoza, ha puesto particular atención en el tema, pues en la región existe un valioso capital patrimonial afectado por esta patología. Así se ha diseñado y construido instrumental, técnicas y procesos de ensayos novedosos para evaluar el ascenso capilar en probetas a escala natural y reducida de adobe. En este trabajo se presenta un procedimiento de ensayo, y el dispositivo correspondiente, para evaluar el comportamiento de una probeta de suelo sometida a ciclos de humedad-secamiento.

\section{Dispositivo de ensayo}

Este ensayo tiene por objetivo la evaluación del agua capilar en su proceso de ascenso y se- 
cado en muestras que se disgregan fácilmente en contacto con agua (arenas finas limo-arcillosas). Este ensayo utiliza determinaciones gravimétricas y fotográficas, empleando equipos económicos en comparación con otros procedimientos que permiten obtener las mismas cuantificaciones pero con equipos sensiblemente más costosos: espectrógrafo nuclear, absorción de rayos gamma, propiedades termales del suelo sobre el contenido de humedad, ondas ultrasónicas, ondas de radar, propiedades dieléctricas.

En el siguiente esquema se muestra el dispositivo de ensayo diseñado, simple y con materiales accesibles y baratos.

El dispositivo de ensayo (Figura 1) consiste en una base porosa, compuesta por una placa de piedra volcánica (2) (denominada comúnmente pómez, $\delta_{\text {aparente }}=0,67 \mathrm{~g} / \mathrm{cm}^{3}$ ) enmarcada por un cuadro o marco (3) de latón (bronce) sostenido por 2 apoyos transversales (8) del mismo material y una hoja de papel poroso (4) $\left(80 \mathrm{~g} / \mathrm{m}^{2}\right)$ que cubre la placa.

Esta base se sumerge parcialmente en una pileta con agua (5), manteniendo su nivel constante (7) (levemente por debajo de la base de la muestra). El marco metálico, conformado en sección " $Z$ ", sumado a la elevación que proveen los apoyos transversales (8), permite el franco ingreso de agua por la parte inferior de la base, la cual es transferida a la probeta (1) a través del papel filtro, evitando que la muestra tome contacto di-

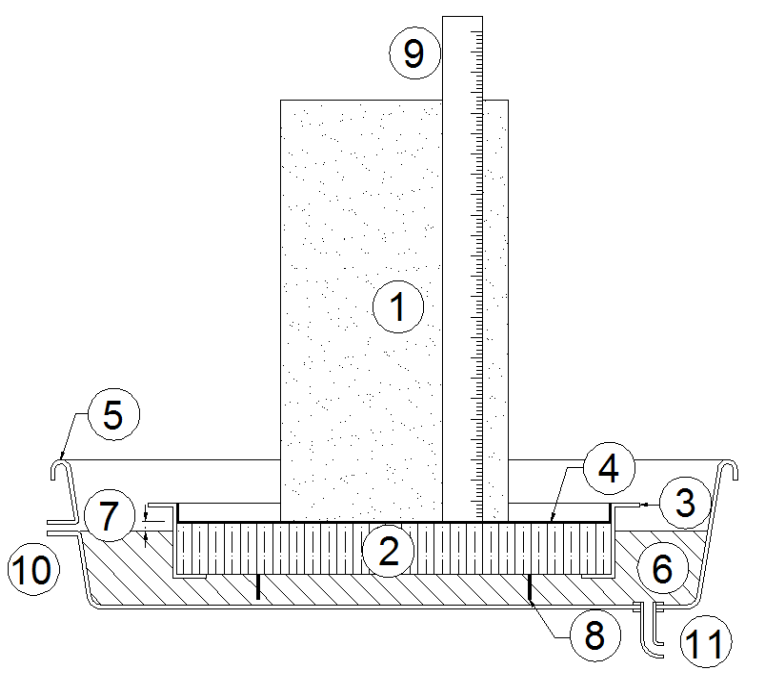

recto con el agua, evitándose así su disgregación (Figura 2).

\section{Procedimiento de ensayo}

El ensayo presenta dos fases: humedecimiento y secado, lo cual constituye un ciclo, pudiéndose realizar varios ciclos, en los cuales se determina cantidad de agua absorbida-perdida por la muestra, variación del grado de humedad media, de altura capilar y la velocidad media e instantánea de ascensión capilar.

Las probetas empleadas se tallan a partir de muestras que pueden provenir de muros existentes (proyectos de restauración) o de prototipos de adobes o tapias que se emplearán luego en mamposterías.

En el segundo caso es posible modificar los valores de las variables que controlan la permeabilidad del suelo (tales como la granulometría, compacidad, plasticidad, etc.) para obtener relaciones óptimas. Además, en muestras donde se han incorporado aditivos (emulsiones asfálticas, cal, cemento, siloxano, gomas, etc.) el ensayo ayuda a establecer el tipo y dosificación en relación con la efectividad observada para reducir o suprimir el ascenso capilar.

El ensayo es particularmente útil en probetas extraídas de muros existentes (muestras inalteradas), pues el ambiente controlado en que se realiza permite evaluar ajustadamente la efectivi-

\begin{tabular}{ll} 
& \multicolumn{1}{c}{ REFERENCIAS } \\
1 & Muestra (probeta) \\
2 & $\begin{array}{l}\text { Placa de piedra volcánica (pómez). } \\
\text { Dimensiones: } 90 \times 220 x \text { alto20mm }\end{array}$ \\
3 & Marco base porosa (de latón) \\
4 & Papel filtro \\
5 & Recipiente contenedor (pileta) \\
6 & Agua \\
7 & Revancha entre el apoyo de la \\
8 & muestra y el nivel de agua (4mm) \\
9 & Regla graduada. \\
10 & Vertedero para derrame por sobre-nivel. \\
11 & Ingreso de agua
\end{tabular}

Figura 1. Esquema del dispositivo. 
dad hidrófuga de un proyecto de intervención y restauración.

\subsection{Preparación de la muestra}

Para este trabajo, las probetas se tallaron a partir de muestras provenientes de muros existentes, correspondiendo a un suelo areno-arcilloso, tipo SC (IRAM 10509) [3], con dimensiones que fueron de $80 \mathrm{~cm}^{2}$ a $200 \mathrm{~cm}^{2}$ por base y altura variable entre $8 \mathrm{~cm}$ a $40 \mathrm{~cm}$.

Cada muestra se llevó a estufa a $60^{\circ}$ hasta alcanzar peso constante.

\subsection{El equipo}

Se depositó la probeta sobre la base porosa que se encontraba parcialmente sumergida en pileta a nivel de agua constante $( \pm 1,5 \mathrm{~mm})$, quedando así la base de probeta $4 \mathrm{~mm}$ por sobre el nivel de agua (Figuras 3 y 4).

\subsection{Determinaciones}

\section{a. Fase humedecimiento}

El conjunto base porosa-muestra se retira de la pileta, reposa 90 segundos sobre un paño humedecido ( $\sin$ superficie brillante), se pesa y luego, retorna a la pileta. Así se continúa, hasta verificar una diferencia entre dos pesadas consecutivas inferior al $2 \%$.

Simultáneamente, se lleva un registro fotográfico del ascenso de la interfase seco-húmedo.

A partir del registro fotográfico puede verse el ascenso en una probeta (Figura 5), se obtiene:

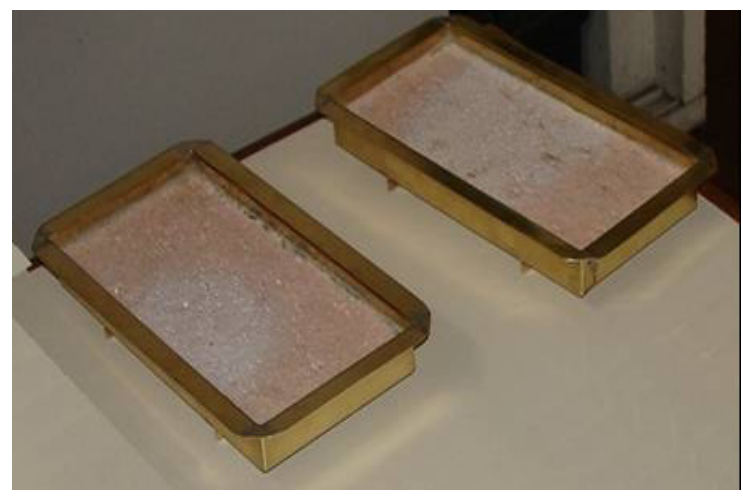

Figura 2. Base porosa (sin papel filtro).

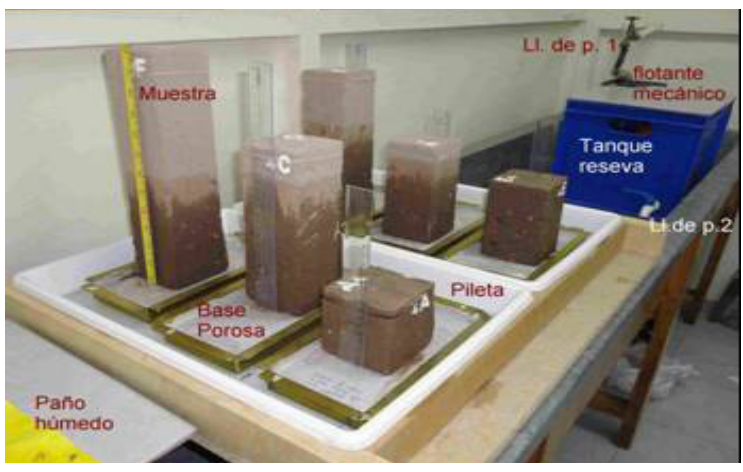

Figura 3. Vista del montaje del equipo.

- La variación de altura capilar en función del tiempo.

- La velocidad media e instantánea de ascenso capilar.

\section{b. Fase secado}

Finalizada la fase de humedecimiento, la probeta se retira de su base porosa y se lleva a secado natural: se deposita sobre una mesa ubicada

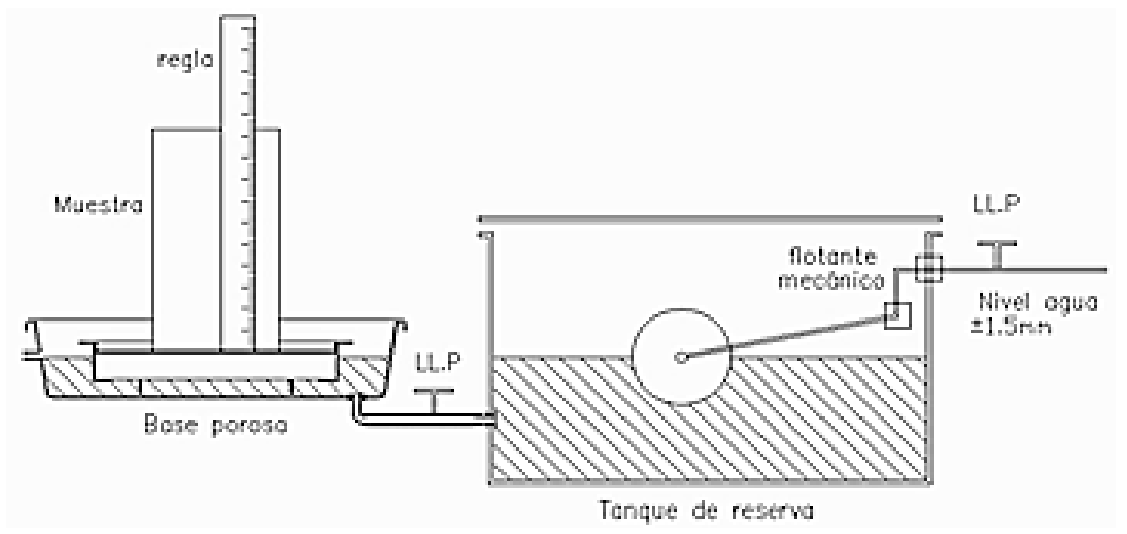

Figura 4. Esquema ensayo. 


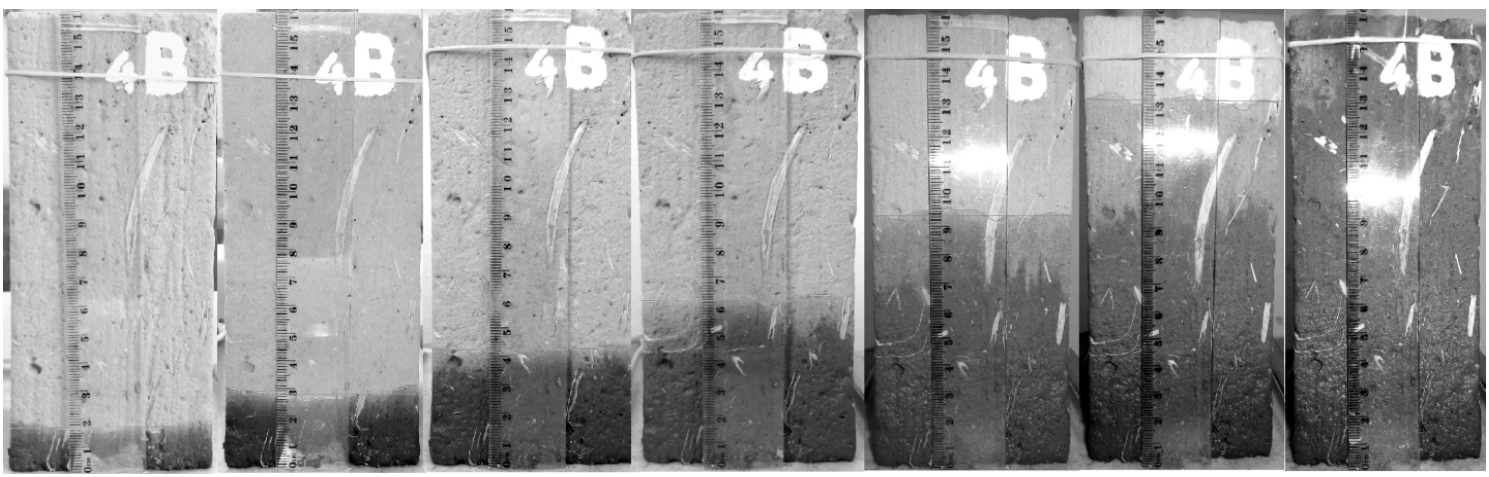

Figura 5. Secuencia fotográfica del proceso de humedecimiento.

al aire libre, bajo techo para evitar el asoleamiento directo y la lluvia. Se registra la variación de peso en el tiempo, hasta que la diferencia entre dos pesadas consecutivas sea inferior al $2 \%$.

El secado comienza en la superficie de la muestra y luego progresivamente continúa hacia el núcleo de la misma [4], razón por la cual sobre la superficie de la probeta no se observa claramente una interfase húmedo-seca. Por ello, en esta fase el registro fotográfico sólo brinda una evaluación cualitativa del proceso de retracción. Según se verá más adelante, el secado puede acelerarse en estufa a $60^{\circ} \mathrm{C}$.

Luego, por diferencias de pesada se determina la variación de:

- Cantidad de agua absorbida-perdida por la muestra.

- La variación del grado de humedad medio, en humectación-secamiento.

\subsection{Discusión de los resultados}

Se practicó el ensayo descripto sobre un total de 60 probetas con las características establecidas en 3.1.

La variación del contenido de humedad en función del tiempo queda definida, para la fase humedecimiento, por una función logarítmica del tipo $\mathrm{w}=\mathrm{a} \cdot \ln (\mathrm{x})+\mathrm{b}$ (Figura 6$)$, donde: $\mathrm{w}$ es el contenido de humedad ( $\mathrm{w}=$ peso de agua acumulada*100/peso suelo seco); $t=$ tiempo; $a=$ coeficiente $1.5<\mathrm{a}<3.5 ; \mathrm{y} \mathrm{b}=$ coeficiente que aumenta con la altura de la probeta.

La Figura 7 muestra los valores de contenido de humedad para dos ciclos de humedecimiento-sequedad para la misma probeta, donde pue-

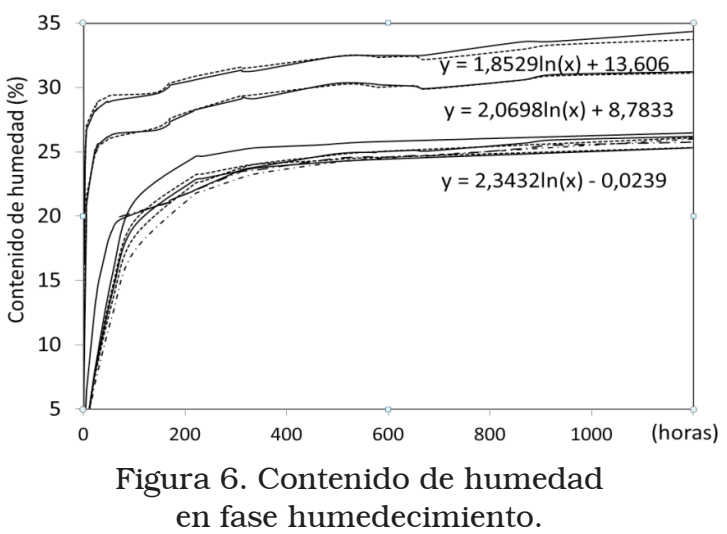

de observarse que las curvas de humedecimiento de ambos ciclos muestran buena correlación $(\mathrm{R}=0.99675)$.

Por su parte las fases de secamiento quedan representadas por una función lineal del tipo $\mathrm{y}=$ - mx +b (Figura 8). Como era de esperar, la pendiente que representa el secado natural es más suave que la correspondiente al secado con estufa, pues seca la probeta unas cuatro veces más rápido. No se observó alteración en la curva de humedecimiento entre probetas secadas al ambiente o en estufa $\left(60^{\circ} \mathrm{C}\right)$.

La velocidad de ascenso capilar queda definida para la fase humedecimiento por una función potencial del tipo $h=a .^{b}$ (Figura 9), en don$\mathrm{de} \mathrm{h}=$ altura de ascenso; $\mathrm{a}=$ coeficiente $2<\mathrm{a}<4.5$; $\mathrm{t}=$ tiempo transcurrido; $\mathrm{y} \mathrm{b}=$ coeficiente $\approx 0.5$.

En todas las determinaciones realizadas durante los ensayos se abarcó importantes intervalos de tiempo, para definir claramente las tendencias que describen el comportamiento. Sin embargo, el ensayo usado como herramienta de trabajo requiere de tiempos sensiblemente meno- 

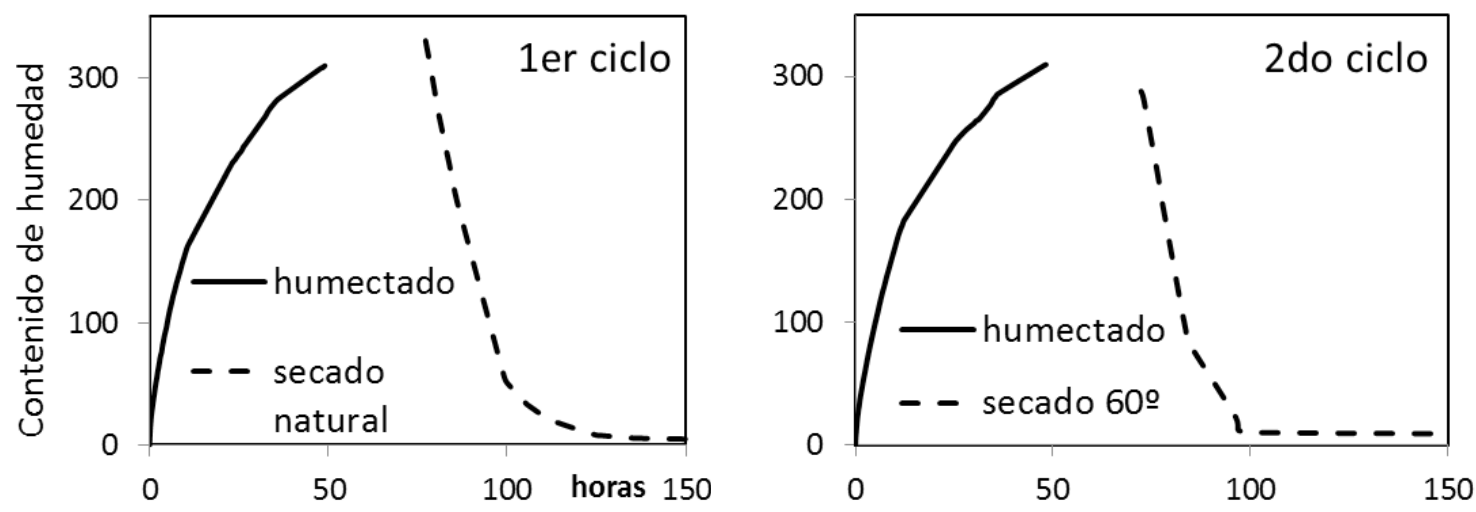

Figura 7. Ciclos de humedecimiento-sequedad.

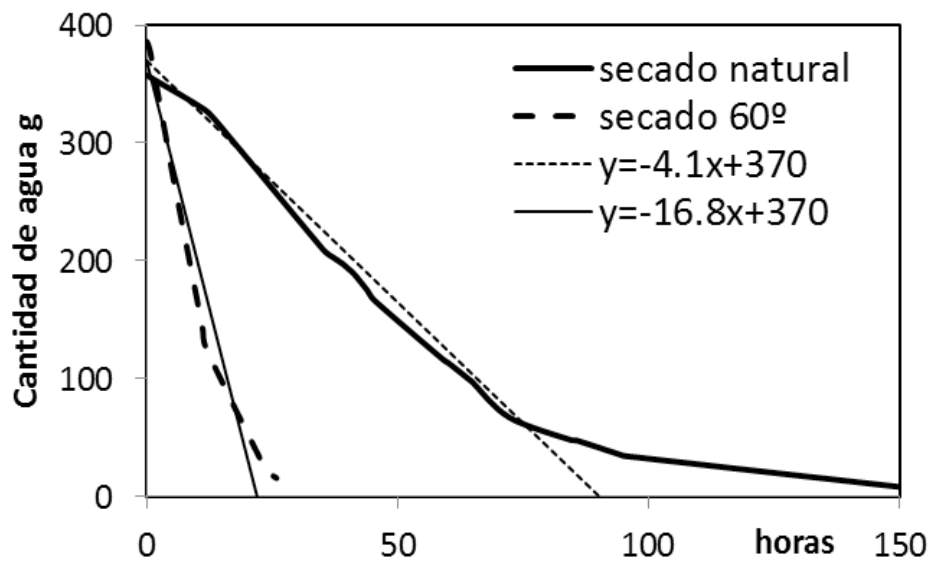

Figura 8. Variaciones entre secado natural y en estufa $\left(60^{\circ} \mathrm{C}\right)$.

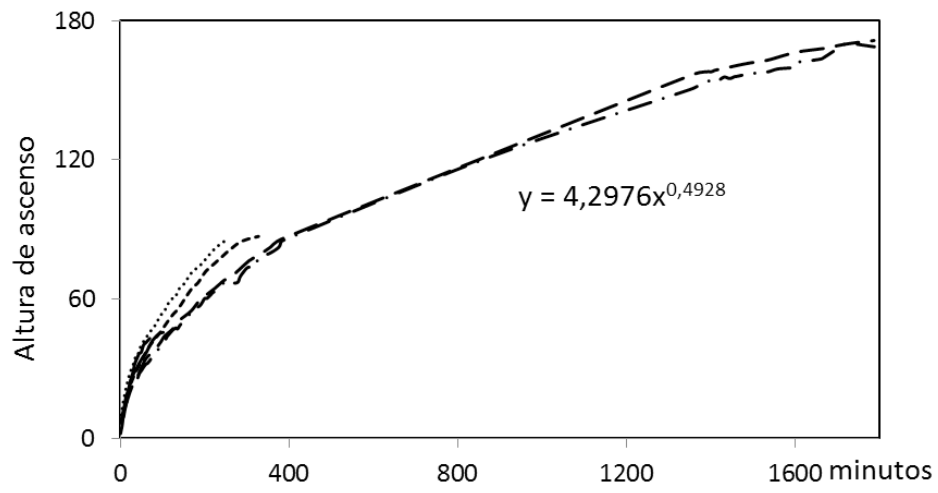

Figura 9. Velocidad de ascenso capilar.

res, en efecto, la fase de humedecimiento queda plenamente descripta su tendencia con un ensayo que dure 24 horas. Mientras que la duración de la fase de secamiento abarcará el lapso entre el inicio del mismo hasta el momento en que la proyección del tramo lineal intercepta a las abscisas (Figura 8).
Se evaluó el grado de perturbación introducido por el retiro-reingreso de base porosa-probeta a pileta, se comparó los valores de altura capilar, con aquellos provenientes de probetas patrón que no fueron retiradas de la pileta. Se halló una alta correlación entre los valores $(\mathrm{R}=0.999994)$, (Figura 10), es decir, el retiro-reingreso no influye. 


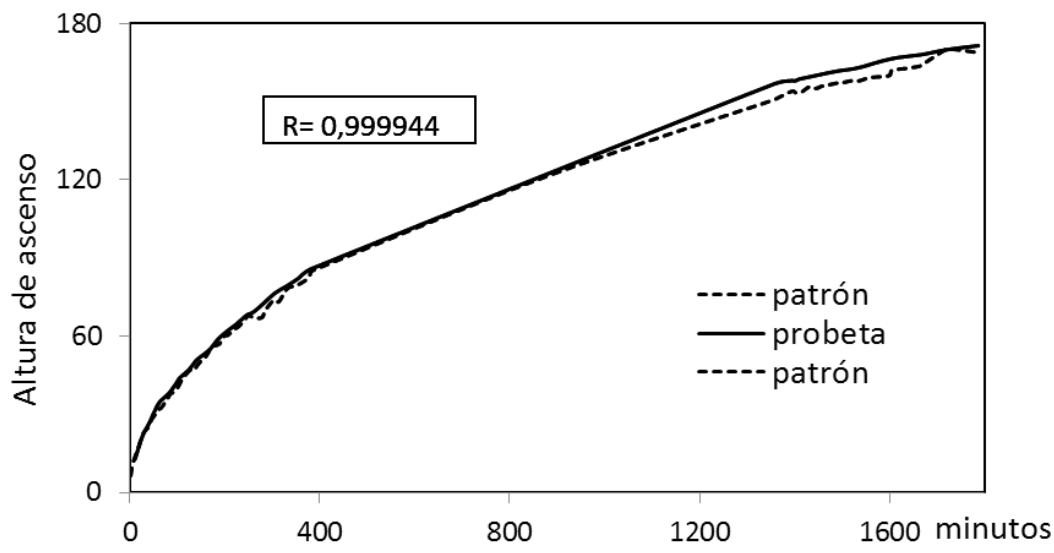

Figura 10. Incidencia del retiro de la probeta-base porosa.

Esta despreciable perturbación en el ensayo se explica porque la tasa de secado en la muestra es 7 veces superior al de la base porosa, por lo tanto, durante el retiro-reposo la misma placa retiene el agua (succión) (Figura 11).

Asimismo, la Figura 6 muestra claramente la reproducibilidad del ensayo que aplicado a probetas de iguales características granulométricas y dimensionales brinda resultados semejantes.

Para concluir, este dispositivo posibilita diseñar procedimientos de consolidación-restauración idóneos, tal que permitan aplicarlos con el pleno conocimiento de su eficiencia, sin poner en riesgo al edificio en estudio debido a una práctica incorrecta [5].

También es de utilidad para probar mezclas con aditivos, o con distintas composiciones granulométricas. Puede aplicarse en obra nueva, para optimizar la relación textura-compacidadaditivos del material usado en la construcción (tapia, adobe), y reducir y/o evaluar la vulnerabilidad al ascenso capilar.

En próximos trabajos se prevé introducir las siguientes modificaciones al ensayo:

1. Parafinar de una a cuatro caras de la muestra para modelar su comportamiento en diversas posiciones de un plano horizontal dentro de la masa del muro.

2. Modificar el espesor de la placa porosa para modelar la influencia de la masa del muro por debajo de la muestra.

3. Evaluar el comportamiento de diferentes tipos de revoques.

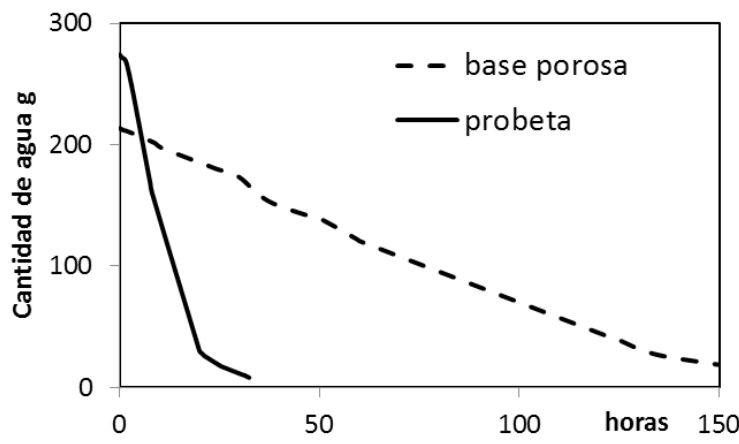

Figura 11. Tasa de secado en probeta y base porosa.

4. Ampliar la escala del ensayo para probetas que permitan representar, la interacción entre el tipo de mampuesto y la argamasa usada. En este caso las probetas tendrían una dimensión de $1 \mathrm{~m}^{2}$ por el ancho del muro en estudio. El significativo peso de la muestra imposibilita la determinación gravimétrica con la precisión necesaria, sin embargo, mediante la fotografía se realiza el seguimiento de la velocidad de ascenso capilar, permitiendo evaluar tipos de texturas, compacidad y aditivos usados. Este ensayo se encuentra en fase de desarrollo.

\section{Conclusiones}

Se ha descripto un procedimiento de ensayo y el dispositivo correspondiente destinado a la evaluación del proceso de ascenso capilar, determinando la velocidad, la variación del agua absorbida, la variación de agua drenada-evaporada, el contenido de humedad y la tasa de secado. 
El trabajo constituye un aporte respecto a otros ensayos semejantes, el dispositivo descripto permite el uso de probetas disgregables en contacto directo con agua (arenas finas limo-arcillosas), mientras que los ensayos estandarizados para evaluar estos efectos se limitan a la absorción superficial de agua en el material y no al proceso de ascenso capilar en sí. Además el procedimiento de ensayo está dirigido específicamente a las mamposterías de adobe y tapia.

Se ha examinado la validez del proceso, chequeado su reproducibilidad y consistencia de resultados.

Los resultados obtenidos permiten valorar la respuesta del material suelo a emplear y así, mejorar su calidad por medio de modificaciones físico-químicas. Asimismo, en el caso de intervenciones estructurales en edificios histórico-patrimoniales, posibilita diseñar procedimientos de consolidación-restauración idóneos.

Por otra parte, el obtener valores controlados de humedad con una distribución variable en vertical, brinda muestras representativas del proceso real para la realización de otros ensayos, por ejemplo, resistencia al corte directo, compresión simple, parámetros mecánicos necesarios para cuantificar la capacidad mecánica del muro en estudio.

La sencillez del ensayo sumada al exiguo valor económico del dispositivo necesario le otorga al procedimiento la posibilidad de difusión en los países en donde se emplean estos materiales constructivos (mayoritariamente países emergen- tes). Por igual razón, el ensayo podría constituir un estándar para clasificar la aptitud de un suelo a usarse en la construcción de mampostería de adobe o tapia.

\section{Referencias bibliográficas}

1. Cirvini, S. y otros. "Patrimonio Arquitectónico del Área Metropolitana de Mendoza. Métodos y Técnicas para su detección, catalogación y evaluación como recurso", ANPCYT - Conicet, Mendoza, 2009. http://www.ahter.org/cd.php

2. CraTerre-AEG, Gci e Iccrom, "Sumary Report, Project Terra Research Meeting”, Torquay, England, (2000). (Preprint)

3. Instituto Argentino de Normalización y Certificación, "Normas IRAM". Las normas utilizadas fueron: 1520; 1533; 45063; 12588; $11561 ; 1590$, cuya consulta se gestiona desde el sitio oficial: http://www.iram.org.ar/index. php?IDM $=28 \& \mathrm{mpal}=5 \&$ alias $=$ Busqueda-deNormas [Consulta: 20 oct.2009- 30 mar.2010]

4. Schroeder, H. "Moisture transfer and change in strength during the construction of earthen buildings". Informes de la Construcción, Vol. 63, N523 (2011) 107-116.

5. Monjo Carrió, J. “Tratado de Rehabilitación. Tomo 3: Patología y técnicas de intervención. Elementos estructurales". Ed. Munilla-Lería, Madrid, 1998.

Recibido el 10 de Diciembre de 2012 En forma revisada el 20 de Enero de 2014 